\title{
Si può anche imprestare qualcosa, ma prestateci attenzione!
}

\author{
Vittorio Coletti
}

PUBBLICATO: 25 GIUGNO 2019

\section{Quesito:}

Sono pervenute molte domande relative al verbo imprestare, se si può considerare equivalente di prestare e se è corretto oppure no.

\section{Si può anche imprestare qualcosa, ma prestateci attenzione!}

$P$

restare e imprestare sono, come è intuibile, parenti stretti e il secondo potrebbe essere, come in genere si sostiene, un derivato del primo col prefisso in-rafforzativo, anche se un impraestare sarebbe attestato, secondo il celebre glossario del Du Cange (Charles Du Cange, Glossarium mediae et infimae Latinitatis, I678), già nel latino medievale accanto a praestare (ma la citazione viene da una carta riportata di seconda mano in un'opera secentesca!). Il Du Cange lo definisce come sinonimo di praestare 'fare un prestito', e, documenti alla mano, anche di commodare 'dare in comodato', diremmo oggi. Comunque sia, i due verbi procedono in coppia, anche se imprestare è attestato in italiano agli inizi del ' 300 , un secolo dopo le prime testimonianze di prestare (I2II). Una coppia simile è quella di prestito e imprestito. Sempre nel XIV secolo, infatti, come si vede dal corpus OVI, accanto al sostantivo prestito ha fatto la sua comparsa in Italia imprestito, attestato pure nella variante impresto e, in documenti non toscani, specie veneti, nel plurale imprestedi (una Camera degli imprestedi o imprestiti fu istituita a Venezia già nel XII secolo per gestire il prestito pubblico). A parte che, sempre secondo il Du Cange, il latino medievale attesta già anche un impraestitum ("facere impraestitum") nel XIII secolo, e che quindi la forma italiana potrebbe esserne un derivato diretto, qui, oltre la plausibile derivazione da prestito con prefisso intensivo (però non confortata dalle datazioni attualmente disponibili), potrebbe trattarsi di una nominalizzazione della frequente collocazione "(dare/prendere/avere) in + prestito", magari avvenuta già nel latino medievale.

Passiamo al significato. Imprestare e imprestito hanno significato sostanzialmente analogo a quello di prestare e prestito. O meglio: se l'oggi desueto (nella lingua comune, sopravvive nel lessico speciale di alcuni linguisti che continuano ad usarlo col valore di prestito, per riferirsi ai forestierismi) imprestito copre, sia pur con minor fortuna, tutta l'area semantica del prestito (e in passato lo ha fatto ancora di più), il pur ancora attuale imprestare non fa altrettanto con quella di prestare, di cui è sinonimo solo per il senso di 'dare in prestito' e non per quello di 'concedere, dare qualcosa di utile, gradito ecc.', come in prestare ascolto, attenzione, aiuto, soccorso ecc.

Dunque prima risposta: prestare e imprestare sono sinonimi nel significato di dare qualcosa in prestito'. E solo in questo. L'àmbito d'uso di imprestare è però (seconda risposta) classificato dai dizionari come meno formale di quello di prestare, più popolare o familiare e dunque corretto ma poco accurato. Per la verità, la storia della parola non autorizzerebbe a confinarla in àmbito esclusivamente popolare, visto che, in passato, è documentata anche in testi di qualche formalità, come si può vedere dalla documentazione del GDLI e da quella reperibile in Google Libri. Forse sarebbe più preciso limitarla ad usi regionali, come si nota dalle sue (poche) attestazioni nei moderni scrittori del Primo Tesoro della Lingua Letteraria Italiana del Novecento a cura di Tullio De Mauro, autori in maggioranza di area settentrionale, non toscana (Calvino, Pavese, Fenoglio, Bassani, Soldati, Ginzburg, Primo Levi, ma anche il romano Moravia). In ogni caso oggi imprestare è sceso o percepito 
a un livello più familiare e informale di prestare e non è un caso se non compare in nessuno dei numerosi e autorevoli, molto formali, testi giuridici (leggi, codici, sentenze) contenuti nel grande corpus BoLC, mentre prestare vi è attestato quasi 800 volte, anche se il numero comprende entrambi i significati fondamentali del verbo ('dare in prestito' e 'concedere') e non solo quello qui in questione. Il maggiore spettro semantico di prestare non permetterebbe, a rigore, di trarre troppe conseguenze dalla sua prevalenza su imprestare nello sterminato deposito di Google, ma le misure sono tali (oltre i 8 milioni contro 35 mila) da consentire di veder ribadita anche su questa piattaforma la superiorità e la preferibilità del verbo semplice, che ha pure il vantaggio di essere più corto.

\section{Cita come:}

Vittorio Coletti, Si può anche imprestare qualcosa, ma prestateci attenzione! , "Italiano digitale", 2019, IX, 2019/2 (aprile-giugno)

DOI: $10.35948 / 2532-9006 / 2020.3127$

Copyright 2019 Accademia della Crusca

Pubblicato con licenza creative commons CC BY-NC-ND 